\title{
Characterizing Swelling Behaviour of Iron Oxides during Solid State Reduction for COREX Application and their Implications on Fines Generation
}

\author{
Taein KANG, ${ }^{11}$ Sushil GUPTA ${ }^{21}$ and Veena SAHAJWALLA ${ }^{21}$ \\ 1) POSCO (Pohang Iron \& Steel Co., Ltd) Works, Pohang, 780-795, Republic of Korea. \\ 2) Sustainable Materials Processing Program, School of Materials Science and Engineering, The University of New South \\ Wales, Sydney, NSW 2052, Australia.
}

(Received on February 16, 2007; accepted on August 24, 2007)

\begin{abstract}
Quality of iron feed source plays a critical role on the efficiency and productivity of advanced ironmaking processes such as COREX. A bench-scale study was conducted to characterize the effect of gas composition on solid state reduction and swelling behaviour of pellets and lumpy state of iron ore for COREX application. Swelling and reduction behaviour of five commercials iron oxides were analysed in a horizontal tube furnace and a thermogravimetric analysis (TGA) reactor respectively by simulating the reducing gas composition and thermal profile of the reduction shaft of COREX process. The degree of reduction and swelling were related to the initial porosity of iron oxide samples. The study demonstrated the strong influence of the initial porosity of iron feed samples on both reduction and swelling characteristics such that high porosity pellets indicated faster reduction and less swelling when compared to low porosity pellets. However, cracks formed in the iron pellets during different stages of reduction could modify the degree of the influence of the initial porosity on the reduction rates due to modification of surface area growth. The compressive strength of samples was found to change non-linearly with progressive reduction such that the crushing strength of pellets declined continuously up to $30 \%$ reduction followed by rapid strength increase as the degree of reduction exceeds $50 \%$ which can be attributed to increasing sintering of metallic phases. The study implies that as far as physical properties are concerned, the initial porosity of iron feed samples can be used to optimize the reduction kinetics, the swelling tendency and the associated implications for the stability of COREX operations including fines generations in the reduction shaft.
\end{abstract}

KEY WORDS: COREX; iron ore; pellet; reduction; swelling; strength.

\section{Introduction}

Increased environmental and economic constrains are driving current blast furnace operation and new ironmaking process such as COREX. The strong effect of iron feed quality on the economic performance of conventional ironmaking blast furnace process is well known. The quality of iron oxides has been extensively studied for conventional blast furnace (BF) or new Direct Reduction (DR) or COREX processes. Despite, some similarity of the new processes with conventional BF process, the understanding of the effect of iron feed quality for the newly developed processes, such as the COREX is still limited and is continuing to further optimize the influence of iron feed quality on the process efficiency. ${ }^{1,2)}$ Due to absence of coke, the requirements of iron feed quality in the COREX reduction shaft is believed to be more stringent as far as the reduction and associated strength characteristics are concerned when compared to BF process. ${ }^{3)}$ Performance of iron source in the COREX process can be influenced by several factors including the physico-chemical properties and the process conditions. These requirements would be similar for Midrex DR plant except that iron oxide reduction predominantly occurs by hydrogen from natural gas, while in a COREX process the reducing gas is predominantly supplied by $\mathrm{CO}$ gas generated due to coal gasification in melter-gasifier section. During indirect reduction, iron oxides expand, crack and often disintegrate to produce fines, which could have serious consequences on the permeability, the productivity and the efficiency. The propensity of fines generation during solid state reduction can be influenced by several factors including physical and chemical properties of iron feed and reaction environment. In past, several studies have been conducted to study the effect of physical properties of iron ore pellets including porosity on the swelling behaviour ${ }^{4-7)}$ and related phenomena such as effect of growth of iron whiskers, ${ }^{8-11)}$ effect of reducing gas composition including the effect of sulfur presence and the temperature, ${ }^{11-16)}$ effect of pelletizing process conditions ${ }^{6,17)}$ and gangue contents. ${ }^{6,7,9-12,17)}$ Iron oxide pellet strength is believed to be related to extent of swelling during reduction. ${ }^{12,18,19)}$ However, the understanding of effect of iron 
feed quality on their performance in commercial COREX operations is far from complete as it is not sufficient to identify the most suitable feed materials.

In the early stages of the COREX development at POSCO, different kinds of iron oxides were tested under similar process conditions in order to demonstrate the highest productivity (2400 hot metal tons/d) with the help of pellets and lumpy ore samples. No successful trials could be completed by using only lumpy ore samples. On the other hand, different pellets indicated different performance and productivity during only pellet operations. Accordingly, quality of iron feed was defined such that the inferior quality samples were found to be associated with reduced permeability, slip and hanging of the burden materials. ${ }^{20-22)}$ High productivity using good quality pellets suggested that sufficient amount of reduction gases were available in the reduction shaft to achieve $90 \%$ metallization. Low metallization in the reduction shaft directly influences the melter gasifier condition, and hence the productivity. Therefore, it was obvious that the impact of iron oxides could be attributed to the differences in their reduction, swelling and subsequent fines generation behavior. However, existing benchscale characterization tests were not sufficient to explain the different performance of various iron oxides samples.

Therefore, an experimental study was conducted to investigate the iron oxides behaviour in a range of reaction conditions under laboratory conditions so that their performance in the reduction shaft of the CORX plant could be better understood. Both chemical and physical properties of iron oxides are known to influence the reduction behaviour. $^{10,23)}$ However, the main objective of this study is limited to investigate the effect of physical properties of iron feed on the reduction and swelling behaviour with particular focus on their implications on COREX operations. The reduction and swelling tests were conducted in a TGA and horizontal tube furnace respectively and were related to the initial porosity of the iron feed samples. Both extent of reduction and swelling measurements were also related to compressive strength variation with progressive degree of reduction.

\section{Experimental}

\subsection{Sample Selection}

Five iron feed samples including three pellets and two lumpy iron ores were used in the initial trials during early stages of the development of the COREX process at POSCO. The same set of samples was selected for this laboratory study mainly due to easy availability as well as previous knowledge of their performance in blast furnace operations. Samples were randomly picked from the stockyard of Pohang works. Since this study was conducted, POSCO has further advanced the COREX process such that the reduction shaft is replaced by fluidized bed in the current operations. However, the reduction shaft is still commonly used to carry out solid state reduction in other COREX operations. In order to minimize the effect of particle size variations, all samples were screened to a narrow size range of 10 to $12 \mathrm{~mm}$. In each tests, sample mass was kept to 3 to $4 \mathrm{~g}$. The chemical and physical analyses of the iron oxide samples are provided in Table 1.
Table 1. Properties of five commercial iron oxide samples.

\begin{tabular}{|l|c|c|c|c|c|}
\hline & \multicolumn{2}{|c|}{ Iron lumps } & \multicolumn{3}{c|}{ Iron pellets } \\
\hline & MTN & ISC & CVR & ALG & SAM \\
\hline $\mathrm{Total} \mathrm{Fe}(\%)$ & 64.49 & 66.30 & 65.86 & 65.92 & 65.84 \\
\hline $\mathrm{SiO}_{2}(\%)$ & 3.53 & 3.11 & 2.11 & 1.95 & 2.54 \\
\hline $\mathrm{Al}_{2} \mathrm{O}_{3}(\%)$ & 1.55 & 1.14 & 0.45 & 0.46 & 0.49 \\
\hline $\mathrm{TiO}_{2}(\%)$ & 0.06 & 0.08 & 0.08 & 0.11 & 0.05 \\
\hline $\mathrm{FeO}(\%)$ & 0.31 & 0.22 & 0.55 & 0.75 & 0.17 \\
\hline $\mathrm{CaO}(\%)$ & 0.03 & 0.08 & 2.36 & 2.34 & 2.16 \\
\hline $\mathrm{MgO}(\%)$ & 0.05 & 0.03 & 0.08 & 0.58 & 0.23 \\
\hline $\mathrm{K}_{2} \mathrm{O}(\%)$ & 0.01 & 0.13 & 0.01 & 0.07 & 0.01 \\
\hline $\mathrm{Na}_{2} \mathrm{O}(\%)$ & 0.01 & 0.02 & 0.01 & 0.04 & 0.02 \\
\hline $\mathrm{Mn}(\%)$ & 0.07 & 0.22 & 0.05 & 0.03 & 0.05 \\
\hline $\mathrm{P}(\%)$ & 0.054 & 0.046 & 0.034 & 0.031 & 0.037 \\
\hline $\mathrm{S}(\%)$ & 0.01 & 0.013 & 0.005 & 0.003 & 0.001 \\
\hline $\mathrm{CaO} /$ SiO & 0.01 & 0.03 & 1.12 & 1.20 & 0.85 \\
\hline $\mathrm{Bulk} \mathrm{density}\left(\mathrm{g} / \mathrm{cm}^{3}\right)$ & 2.70 & 2.29 & 2.24 & 2.14 & 1.84 \\
\hline Porosity $(\%)$ & 6.1 & 9.1 & 22.6 & 24.0 & 29.4 \\
\hline Surface area $\left(\mathrm{m}^{2} / \mathrm{g}\right)$ & 0.55 & 0.66 & 0.73 & 1.59 & 1.25 \\
\hline
\end{tabular}

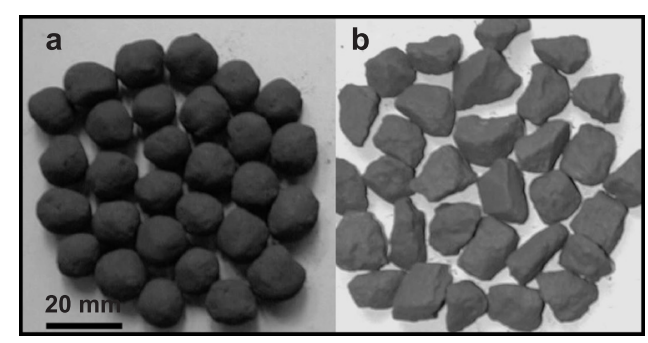

Fig. 1. (a) Physical appearance of the highest porosity SAM pellet and (b) the least porosity MTN lumpy ore.

Bulk density of samples was measured immersion method using $1 \mathrm{~L}$ cylinder with $6 \mathrm{~cm}$ diameter, indicating the highest bulk density of MTN sample. Mercury porosimeter (Autopore 9200) was used to determine porosity. All samples primarily differed in the porosity such that the porosity ( $\sim 20 \%$ ) of the pellets was approximately double that of lumpy samples. BET (Brunauer-Emmett-Teller) surface area of samples was obtained using $\mathrm{N}_{2}$ adsorption. In general, high porosity samples indicated high surface area. Figure 1 illustrates typical morphology of pellets and lump samples of iron ore and shows that pellets are more regular and spherical in shape while lumpy ore samples were irregular with sharp edges. Figure 2 further illustrates typical microstructure of three samples. The highest porosity of SAM pellet and the least porosity of MTN lumpy sample are clearly visible in these images.

\subsection{Reduction Behaviour: Thermogravimetric Analy- sis}

Reduction behaviour of iron oxides was examined in a custom made TGA reactor by continuously monitoring weight loss under controlled reaction environment. Figure 3 is an illustration of the TGA system which consists of $70 \mathrm{~mm}$ outer diameter alumina tube as a reacting zone with bottom loading ability. Weight loss is recorded by a Precisa (1 212 M SCS) analytical balance with accuracy of $\pm 1 \mathrm{mg}$. The extent of reduction was quantified on the basis of weight loss of iron oxide samples in the TGA reactor. Iron oxide specimens were placed in a specially designed Kanthal basket, and suspended by a stainless steel wire from the arm of the balance. Samples were heated to the specified reaction temperature in the presence of nitrogen stream followed by purging with the specified reduction gas. The 


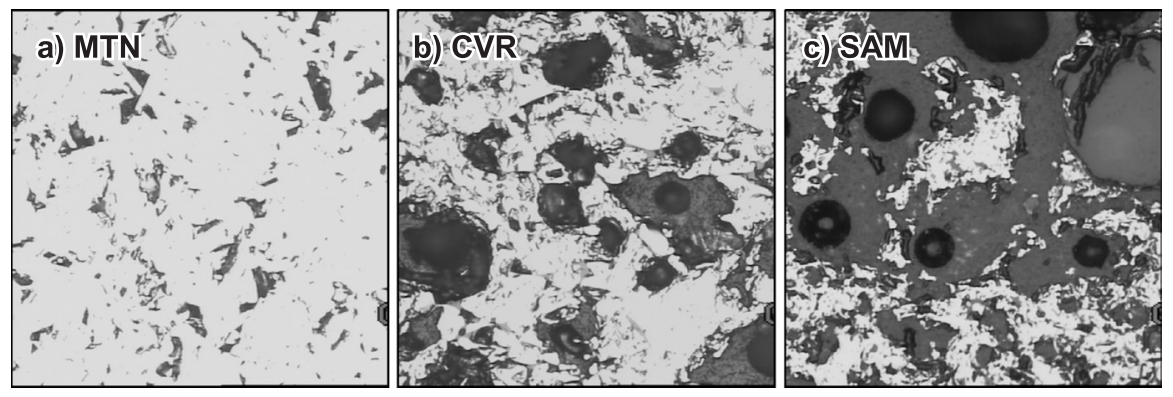

Fig. 2. (a) Microstructure of three iron oxide samples, white regions indicate metal oxide while grey and dark regions show porous phase, (a) least porosity MTN sample, (b) medium porosity CVR pellet and (c) highest porosity SAM pellet.

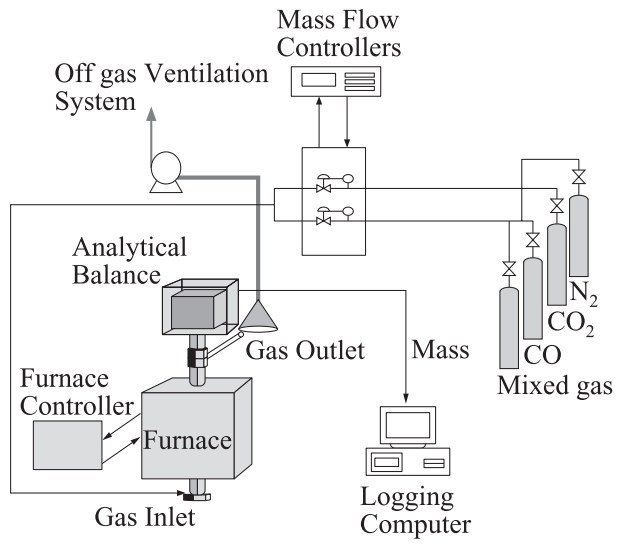

Fig. 3. Schematic of the TGA reactor used for measuring reduction behaviour of iron oxides.

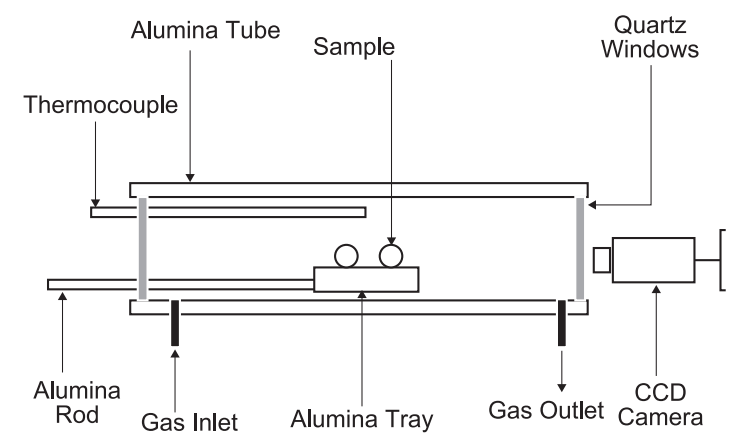

Fig. 4. Schematic of the horizontal furnace used for measuring swelling behaviour of iron oxides.

gas flow rate was always kept sufficiently high enough ( $2 \mathrm{~L} / \mathrm{min})$ to minimize the mass transfer effect. ${ }^{22}$

\subsection{Swelling Behaviour: Horizontal Tube Furnace}

Swelling behaviour of iron oxides was examined by visualizing physical changes of specimen in a custom made horizontal tube furnace. The schematic diagram of the experimental setup is shown in Fig. 4. The internal diameter of the furnace tube was $50 \mathrm{~mm}$. The reaction temperature is achieved by resistance heating of the Super-Kanthal heating element fed with a low voltage-high current power supply. A separate external thermocouple was used to indicate the work tube and reaction zone temperature. The samples were located on a specimen holder which could be pushed to a pre-determined location in the furnace with help of a graphite rod. A CCD camera was used to capture the sample images during reduction. The output from the camera was connected to a VCR to record the entire process as a function of time. A time-date generator was used in the system to display the date and the duration of the process. Images were captured after every $10 \mathrm{~min}$ from the recorded videotape for further processing. An image processing software was used to calculate frontal cross-sectional area and degree of swelling was calculated on the basis of changes in the measured area using Eq. (1).

$$
\begin{aligned}
\text { Degree of swelling } & =\left(\frac{V-V_{\mathrm{o}}}{V_{\mathrm{o}}}\right) \times 100 \\
& =\left[\left(\frac{S}{S_{\mathrm{o}}}\right)^{3 / 2}-1\right] \times 100
\end{aligned}
$$

Where, $V_{0}, V$ are the volumes of sample before and after experiment and $S_{0}, S$ are the corresponding cross-sectional areas. Visual inspection of the specimen images was used to identify the temperature of the crack initiation. Samples were heated up to $1273 \mathrm{~K}$ in the presence of nitrogen atmosphere to confirm that thermal effects did not contribute to specimen expansion or cracking. ${ }^{22)}$

\subsection{Compressive Strength Measurements}

Fines generation behaviour was characterized by measuring compressive strength such that reduction of compressive strength would lead to greater fines generation. Compressive strength of the reacted samples was measured using INSTRON 1185 tester with a cross head speed of $2 \mathrm{~mm} / \mathrm{min}$ at room temperature. Maximum value of the load observed just before an abrupt fall with an extensive fracture of the particle was taken as the compressive/crushing strength. ${ }^{22)}$ Samples with different degrees of reduction were prepared by reacting three particles of different samples with different gases in TGA at $1173 \mathrm{~K}$. Reacted samples were quenched with $\mathrm{N}_{2}$ slowly to room temperature in the TGA. $\mathrm{H}_{2}$ was used to prepare samples of more than $30 \%$ reduction while mixed gases were used to prepare samples of less than $20 \%$ reduction. Due to fast reaction rates of $\mathrm{H}_{2}$, it was not convenient to use $\mathrm{H}_{2}$ to prepare samples will low degree of reduction. Average values of compressive strength measurements were obtained after using repeat runs.

\section{Results and Discussion}

Both iron oxide characteristics and process conditions 
can affect their performance in a reduction shaft. The main focus of this study is to characterize the effect of iron oxide particularly their physical properties. The chemical composition of all the samples was similar such that iron content of all the samples was of the same order being $65 \%$. Basicity of pellets was greater than lumpy ores however, the samples were mainly distinguished on the basis of the differences of physical properties. Figure 5 shows that pellets had relatively greater $\mathrm{Hg}$ intrusion volume compared to lump iron oxides. Porosity of all the pellet samples, as calculated using the mercury intrusion data and material density, was more than $20 \%$ while that of lumpy samples was less than $10 \%$ (Table 1). Average pore diameter of the pellets was also higher $(1.4 \mu \mathrm{m}$ and $7.6 \mu \mathrm{m})$ when compared to those of lumpy samples $(0.07$ to $0.18 \mu \mathrm{m})$. Reduction and swelling tests were conducted in separate furnaces. All efforts were made to simulate similar reaction environments by keeping similar reacting gas composition, time and temperatures.

\subsection{Effect of Hydrogen}

Figure 6(a) compares the isothermal reduction of five samples during reactions with $\mathrm{H}_{2}$ at $1073 \mathrm{~K}$. With hydrogen, all the samples were reduced to more than $90 \%$ in less than $30 \mathrm{~min}$. The highest porosity sample (SAM pellet) in-

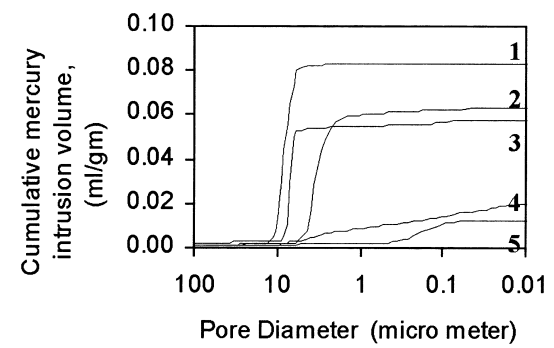

Fig. 5. Cumulative mercury intrusion volumes of SAM (1), ALG (2), CVR (3), ISC (4) and MTN (5) samples plotted against their pore size.
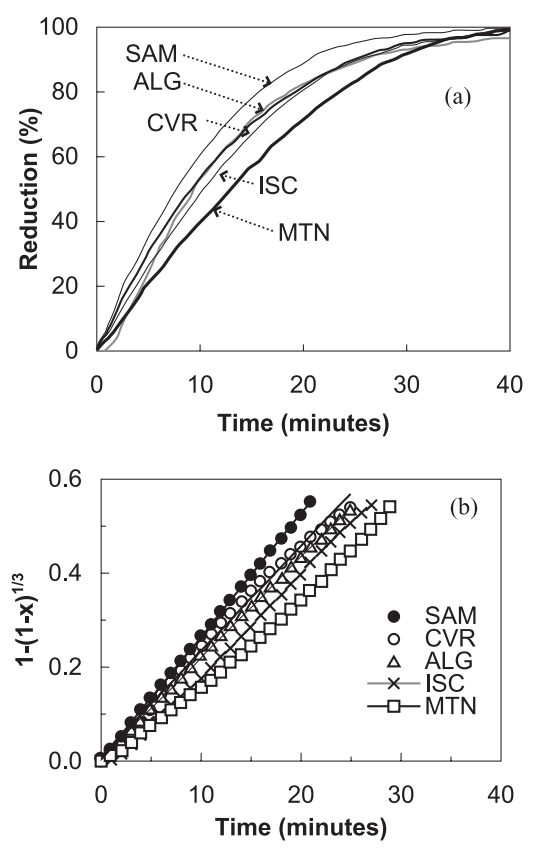

Fig. 6. (a) Weight loss of five iron oxides with time during isothermal reduction with $\mathrm{H}_{2}$ in the TGA reactor at $1073 \mathrm{~K}$ and (b) variation of reduction rates with time. dicated the fastest reduction rate while the least porosity sample (MTN lump) provided the least rates. Reduction of iron oxide by hydrogen is believed to be controlled by the reaction at the oxide-metal interface ${ }^{24-27)}$ such that the reduction kinetics can be expressed by Eq. (2).

$$
\left[1-(1-x)^{1 / 3}\right]=k t+c \text {. }
$$

Where $x$ is the fraction of iron oxide converted at time $t, k$ and $c$ are constants. Figure 6(b) plots the fractional reduction $\left[1-(1-x)^{1 / 3}\right]$ versus times, and indicates a linear correlation for all the samples up to $90 \%$ reduction. Thus, in our experimental conditions, reduction by hydrogen is controlled by the chemically controlled reactions as suggested in a related study. ${ }^{10)}$ Figure 6(a) further shows that reduction rates of samples MTN and SAM marginally decrease after more than $90 \%$ reduction, which can be attributed to increases sintering of reduced metallic phases of iron oxides as suggested in past. ${ }^{28)}$ Figure 7 further clearly illustrates that reduction kinetic is strongly influenced by the initial porosity such that the time required for $50 \%$ reduction decreases from 12 to $8 \mathrm{~min}$ with increasing values of the initial porosity of samples. This trend continued to $90 \%$ reduction. Similar observations were also noted in a related study in which faster reduction was reported low porosity ores. $^{29)}$ It may be noted that in general, the high surface area pellets indicated faster reduction when compared to low surface area lumpy ores, however, no clear correlation could be established between their initial surface areas of all samples and different times requires for different stages of reduction.

The swelling of iron oxides during reduction with hydro-

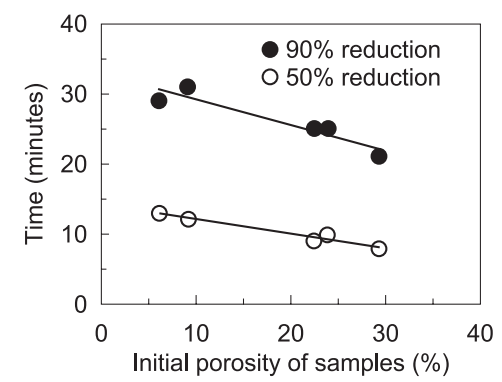

Fig. 7. Correlation between the initial porosity of iron oxides and time required for $50 \%$ weight loss in the TGA reactor during isothermal reduction with $\mathrm{H}_{2}$ at $1073 \mathrm{~K}$.

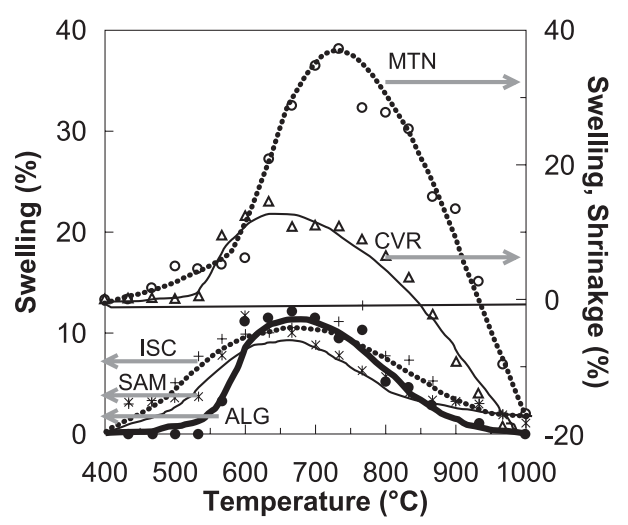

Fig. 8. Comparison of swelling behavior of five iron oxide samples with temperature during reduction with $\mathrm{H}_{2}$ in a horizontal furnace. Swelling and shrinkage changes of MTN and CVR samples are plotted on secondary $Y$-axis. 

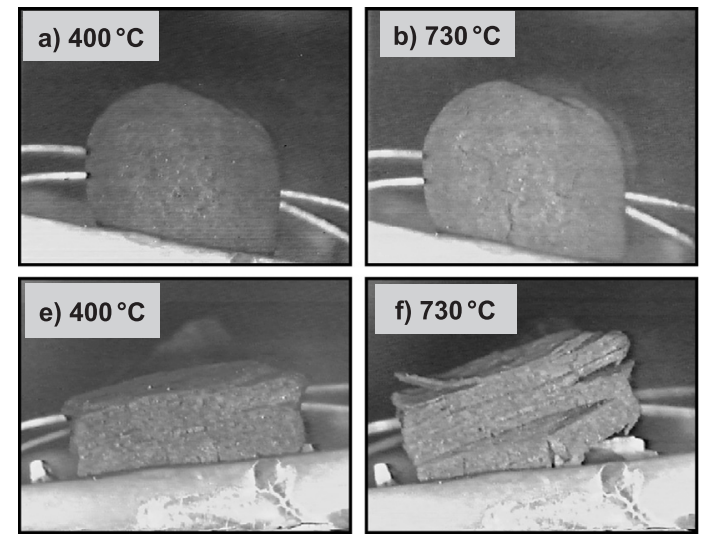
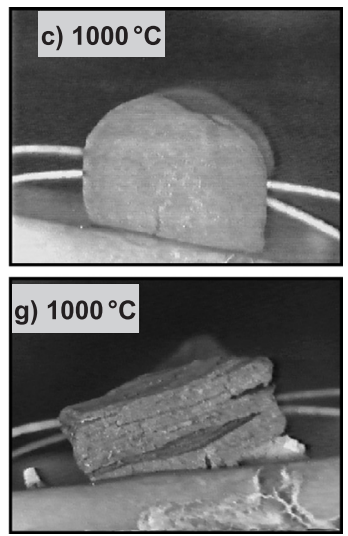

Fig. 9. Illustration of different stages of swelling of CVR pellet at during non-isothermal reduction with $\mathrm{H}_{2}$ i.e. after completion of (a) $15 \mathrm{~s}$, (b) $100 \mathrm{~min}$ and (c) $180 \mathrm{~min}$ of reduction. Approximate temperatures of the samples are indicated in each figure. Similar images of sample MTN are shown in (d) initial stage, (e) maximum swelling and (f) maximum shrinkage.

gen was examined under non-isothermal conditions from 673 to $1273 \mathrm{~K}$ by keeping gas flow rate of $1 \mathrm{~L} / \mathrm{min}$. Figure 8 provides a quantitative comparison of the swelling behaviour of five iron oxides with increasing temperature during reactions with $\mathrm{H}_{2}$ gas. Due to high values of swelling as well as shrinkage at higher temperatures, MTN and CVR samples are plotted along secondary $Y$-axis. Only MTN sample indicated exceptionally high or abnormal swelling while other samples displayed less than $20 \%$ swelling which is generally considered to be normal swelling. Shrinkage of MTN and CVR pellets can be attributed to sintering of metallic phases after significant reduction as discussed before.

Figure 9 illustrates swelling of a pellet and a lumpy ore sample at different stages of reduction by $\mathrm{H}_{2}$. Figure 9(a) shows the initial state of the pellet (CVR) after $15 \mathrm{~s}$ when the sample temperature was about $673 \mathrm{~K}$ while Fig. 9(b) show the swelling after $100 \mathrm{~min}$ of reduction at which the temperature approaches to about $1003 \mathrm{~K}$. Figure 9(c) indicates the final state of shrinkage after $180 \mathrm{~min}$ when the temperature becomes about $1273 \mathrm{~K}$. Figures 9(d), 9(e) and 9(f) illustrates different stages of MTN lumpy sample after undergoing through similar time and temperature profile. Figure 9(d) shows the initial state of sample MTN at the commencement of reduction. Figure 9(b) illustrates the maximum swelling of sample MTN which occurred after $100 \mathrm{~min}$. Maximum shrinkage state of sample MTN noted in Fig. 8 is illustrated in Fig. 9(f). It may be noted that abnormal swelling was only indicated by MTN sample which also had the least porosity.

During swelling, all samples were also accompanied by crack initiation. The temperature range of crack initiation of all samples was estimated on the basis of image analysis, as shown in Fig. 10. In order to minimize the uncertainties associated with visual observation, average values of repeat runs were used. According to Fig. 10, lumpy iron ores appear to begin cracking at relatively higher temperatures compared to the pellet samples. However, no clear relationship between crack initiation temperatures and the initial porosity of samples could be established as the repeatability of these temperatures was within $\pm 25^{\circ} \mathrm{C}$. Figure 10 also shows that all iron oxides displayed crack initiation in a temperature range of $773 \mathrm{~K}$ and $923 \mathrm{~K}$ i.e. after 30 to

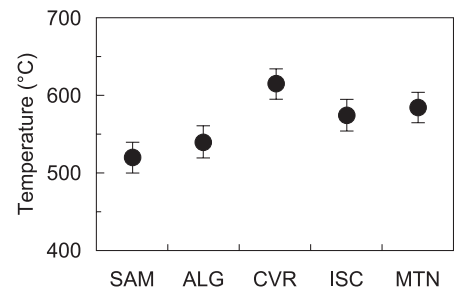

Fig. 10. Temperature range of crack initiation of five iron oxide samples during non-isothermal reduction with $\mathrm{H}_{2}$. This temperature range also corresponds to time interval of 30 to $75 \mathrm{~min}$ as samples were uniformly heated.

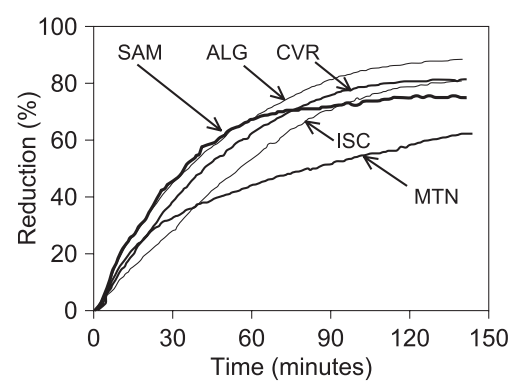

Fig. 11. Comparison of reduction behaviour of five iron oxides during isothermal reduction with melter-gasifier gases $\left(90 \% \mathrm{CO} / 10 \% \mathrm{CO}_{2}\right)$ at $1073 \mathrm{~K}$ in a TGA reactor.

75 min of the beginning of the reduction reactions.

\subsection{Effect of Melter-Gasifier Gases}

The $\mathrm{CO}$ and $\mathrm{CO}_{2}$ are the main constituents of typical product gases formed after combustion and gasification of coal in the melter-gasifier section of a COREX plant. The product gases are rich in carbon monoxide such that $\mathrm{CO} / \mathrm{CO}_{2}$ ratio of the reducing gas entering the reduction shaft would be around 90/10. The temperature of these reducing gases is generally maintained at less $1173 \mathrm{~K}$ in order to allow maximum reduction as well as to avoid sticking or sintering in the reduction shaft. Therefore, in order to simulate reaction environment of lower part of the reduction shaft, a gas mixture of $90 \% \mathrm{CO}$ and $10 \% \mathrm{CO}_{2}$ gases was introduced in the TGA reactor at $1173 \mathrm{~K}$.

Figure 11 compares the reduction of iron oxides with melter-gasifier gases at $1173 \mathrm{~K}$. In general the reduction of 
high porosity samples was greater than lower porosity samples except the reduction behaviour of SAM pellet which did not show this trend after $60 \%$ reduction despite being highest porosity sample. As expected, both high porosity samples SAM and ALG pellets displayed the fastest reduction rate at least up to $68 \%$ reduction which could be attributed to highest values of initial surface areas being $1.6 \mathrm{~m}^{2} / \mathrm{g}$ and $1.25 \mathrm{~m}^{2} / \mathrm{g}$ respectively. However, after $140 \mathrm{~min}$, the ALG pellet displayed almost $90 \%$ reduction and CVR and ISC were reduced to about $80 \%$. MTN sample indicated the least degree of final reduction i.e. $62 \%$. Highest rate and degree of reduction of ALG pellet could be related to highest initial surface area which continued to grow up to final stage of reduction.

Figure 11 further shows that the reduction rate of the SAM pellet, highest porosity sample, decreases after $68 \%$ reduction, while the reduction rate of $\mathrm{MTN}$, the least porosity sample, declined approximately after 30\% reduction. Thus, both SAM pellet and MTN lumpy ore samples indicated a similar trend of declining reduction rates once they exceed certain degrees of reduction levels. This behavior of decreasing reduction rates of SAM and MTN samples were repeatedly observed in different test runs. ${ }^{22)}$ Surface area of an iron oxide is expected to increase with increasing cracks. Consequently, the reduction rate of cracked samples could be greater than the non-cracked sample. It may be noted, that during reduction, sample SAM did not indicate any cracks, which were generally noted for the rest of the samples. Therefore, initial rapid reduction of SAM could be attributed to its initial high porosity and relatively higher sur-

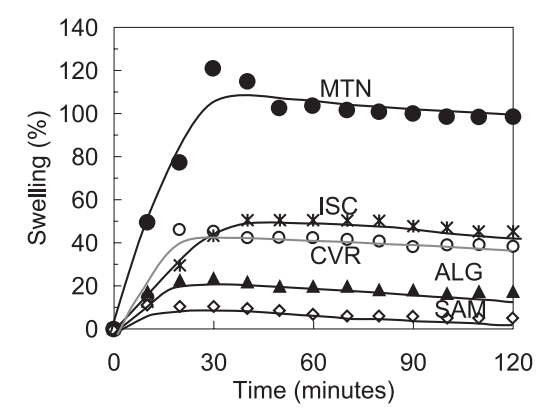

Fig. 12. Comparison of swelling of iron oxide samples with time during isothermal reduction with melter-gasifier gases $\left(90 \% \mathrm{CO} / 10 \% \mathrm{CO}_{2}\right)$ at $1173 \mathrm{~K}$ in a horizontal furnace. face area, while retardation after $68 \%$ could be related to insufficient growth of surface area due to absence of cracks. A similar argument can be used to explain the reduction of MTN sample such that despite lower surface, its higher reduction rates in the initial stage of reduction can be attributed to big cracks generated before $30 \%$ reduction. Thus in general, high porosity samples, which also often have higher surface area, would provide higher reduction rate. However, during different stages of reduction, surface area growth can be modified as a consequence of the characteristics of cracks generated, and trend is consistent with previous studies. $^{24,28)}$ Thus, even though final reduction level of MTN is low when compared to other samples, due to rapid crack generation, it could be reduced rapidly at least up to initial $30 \%$ reduction, which occurred in first $30 \mathrm{~min}$.

Figure 12 shows that after initial $30 \mathrm{~min}$ of reduction, MTN recorded up to $120 \%$ swelling and followed by volume reduction, which was maintained at $100 \%$ swelling level. The CVR and ISC samples showed approximately maximum swelling of $50 \%$ followed by $5 \%$ reduction of swelling at later stages of reduction. ALG and SAM showed the normal swelling during the reduction.

Figure 13 illustrates different swelling states of the least porous lumpy ore (MTN) and the highest porous pellet (SAM) at different time intervals of reduction. All the samples showed crack formation after 5 to $15 \mathrm{~min}$ except sample SAM which did not indicate any noticeable crack in any stage of reduction as seen in Figs. 13(a), 13(b) and 13(c). Figure 13(e) clearly shows the presence of large cracks after $30 \mathrm{~min}$ of reduction, which would also contribute for faster reduction during initial stage of reduction (Fig. 11).

\subsection{Effect of Melter-Gasifier Exit Gases}

Typical COREX exit gases comprise of $36 \% \mathrm{CO}, 32 \%$ $\mathrm{CO}_{2}, 16 \% \mathrm{H}_{2}$ and $16 \% \mathrm{~N}_{2}$. For the sake of convenience, hereafter this composition will be referred as 'mixed gas' or 'Corex exit gas'. Mixed gases were used in order to estimate the possible extent of reduction in the top regions of the reduction shaft. In this study, the reducing temperature of gases was maintained at $1173 \mathrm{~K}$ in order to avoid carbon deposition, even though the actual operating temperatures of the top section of the reduction shaft of the plant is often relatively less.

Figure 14 shows the clear differences of reduction be-
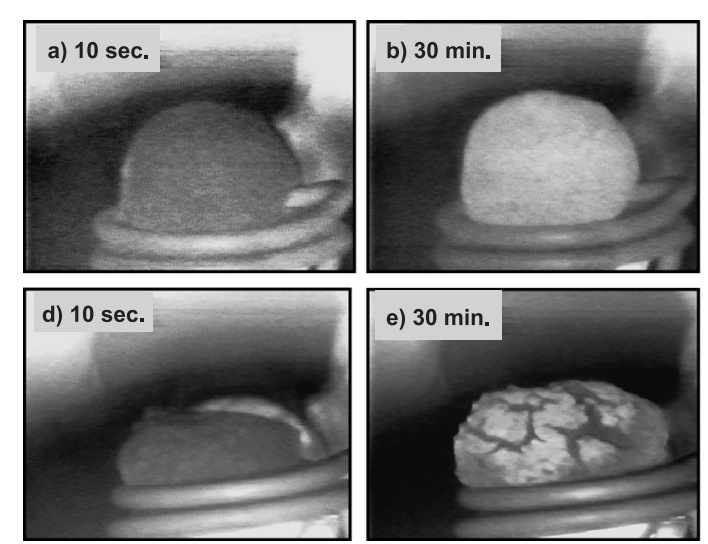
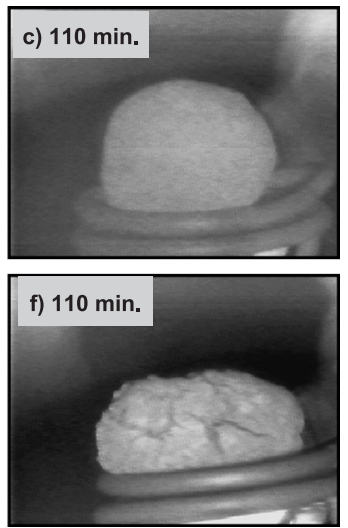

Fig. 13. Illustration of different stages of swelling of the highest porosity SAM pellet sample (a, b, c) and the least porosity MTN lumpy sample $(\mathrm{d}, \mathrm{e}, \mathrm{f})$ at different time intervals during isothermal reduction with melter-gasifier gases $\left(90 \% \mathrm{CO} / 10 \% \mathrm{CO}_{2}\right)$ at $1173 \mathrm{~K}$ in a horizontal furnace. 


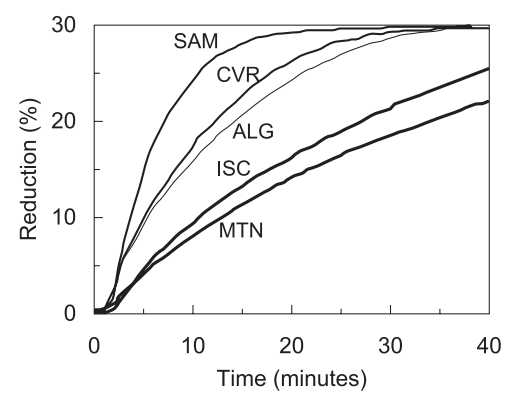

Fig. 14. Comparison of reduction behaviour of five iron oxide samples with time during isothermal reduction with melter-gasifier exit gases or mix gases $(36 \% \mathrm{CO}, 32 \%$ $\mathrm{CO}_{2}, 16 \% \mathrm{H}_{2}, 16 \% \mathrm{~N}_{2}$ ) at $1173 \mathrm{~K}$ in a TGA reactor.

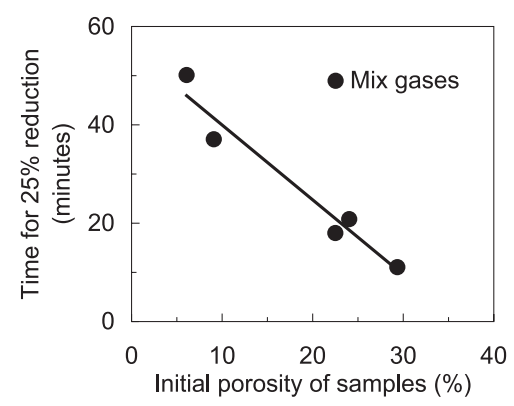

Fig. 15. Correlation between the initial porosity of five iron oxides and the time required for $25 \%$ reduction during isothermal reduction with melter-gasifier exit gases/ mixed gas at $1173 \mathrm{~K}$ in a TGA reactor.

haviour of the pellet and lumpy samples by the mixed gas. The pellet samples approached a steady value of $30 \%$ reduction in about $35 \mathrm{~min}$. For the same period, lumpy samples indicated relatively lower reduction rates however the reduction reaction did not slow down. Figure 14 clearly show that, the reduction behaviour of iron oxides with COREX exit gases was consistently influenced by the initial porosity of the samples. Figure 15 clearly shows that high porosity samples required less time to achieve $25 \%$ of reduction compared to low porosity samples.

Comparison of final degrees of reduction by different various gases indicate that mix gases provide less degree of final reduction which can be explained on the basis of composition of the mix gases. During reaction with $53 \% \mathrm{CO}$ and $47 \% \mathrm{CO}_{2}$ at $1173 \mathrm{~K}$ i.e. the $\mathrm{CO} / \mathrm{CO}_{2}$ ratio of the mixed gases used, wustite is the only possible stable phase of iron oxide. Accordingly, if the contribution by hydrogen reduction is negligible, with this composition of reducing gases i.e. mixed gases, maximum $33 \%$ of reduction is expected. Moreover, due to lower temperatures and potential impact of carbon deposition, the reduction rate in the reduction shaft of an operating COREX plant could be even less than that noted in the TGA reactor i.e. $33 \%$.

In order to study swelling and crack formation in the upper part of the reduction shaft, iron oxides were reacted with mixed gas by heating at the rate of $423 \mathrm{~K}$ per hour from 773 to $1073 \mathrm{~K}$. Figure 16 compares the swelling behaviour of five iron oxides during reaction with mix gases. Swelling trend was similar to that observed during reduction with melter-gasifier gases such that MTN provided the highest maximum swelling $(100 \%)$ and followed by ISC (41\%) and CVR (32\%). Swelling of ALG and SAM was

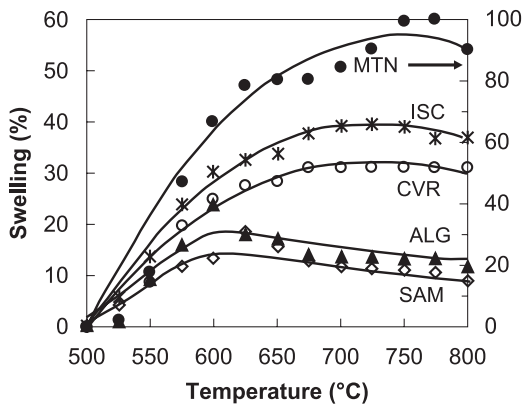

Fig. 16. Comparison of swelling of iron oxide samples with temperature during non-isothermal reduction with meltergasifier exit gases in a horizontal furnace. Swelling data of MTN sample is plotted on secondary $Y$-axis.

less than $20 \%$ and considered to be normal, and was similar to that seen during reaction with melter-gasifier gases. All samples indicated large cracks after 15 to 30 min except ALG and SAM samples. ${ }^{22)}$

\subsection{Implications of Reduction and Swelling Behaviour on Fines Generation}

Due to the absence of low density coke material in the COREX reduction shaft, the properties of iron oxides needs to be much stronger compared to blast furnace iron feed samples. ${ }^{3)}$ Fines generation of iron oxides are believed to affect the permeability of the reduction shaft, and will be dependent on the modification of its swelling, reducing, strength as these are progressively moved from upper zones of reduction shaft towards the melter-gasifier section. Iron oxide strength and hence their potential to generate fines can be compared by measuring the compressive strength of samples after reducing to different degrees.

MTN lump indicated much higher swelling index compared to other samples. During initial POSCO trials, MTN lumpy ore could never be successfully used. Whenever, MTN sample was charged to the furnace, the gas permeability of the burden in the reduction shaft decreased dramatically due to swelling of iron ore. Thus, current observation of the highest swelling of MTN sample in the present study is consistent with the most difficult operating experience when tested in previous plant trials. It is interesting to note that the swelling patterns of five iron oxide samples during reduction with mixed gas is similar to that displayed during reduction with melter-gasifier gases despite the big difference in final reduction degrees. With melter-gasifier gases, all samples could be reduced to more than $65 \%$, while with mix gases all samples could be reduced up to approximately $25 \%$ with the exception of sample MTN which could be reduced only up to $15 \%$. This result implies that the swelling and the cracking behaviour would influence the reduction behaviour of iron oxides in upper part of the reduction shaft.

Figure 17 shows the relationship between maximum swelling and initial porosity. Figure 17 clearly demonstrates the strong effect of the porosity in determining their swelling behavior. In a related study, high porosity was related low swelling due to accommodation of stresses induced during reduction. ${ }^{4)}$ Smaller porosity provided larger swelling (Fig. 17) and less reduction rates (Fig. 7) for both reducing gases of melter-gasifier and those of exit gases. 


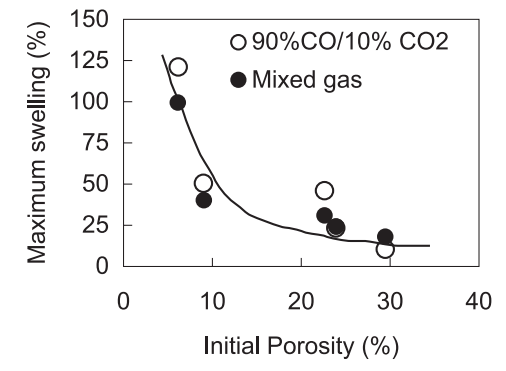

Fig. 17. Correlation between maximum swelling and the initial porosity of iron oxides samples during reactions with different type of reacting gases.

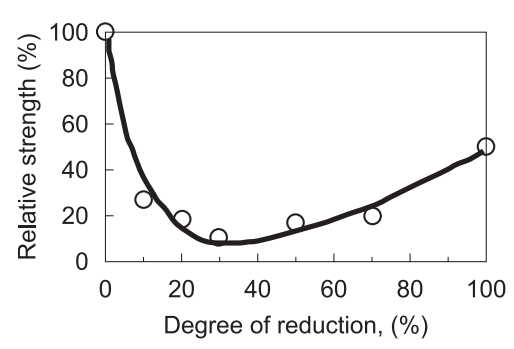

Fig. 18. Correlation of relative compressive strength variation of iron oxides samples with progressive reduction in a TGA reactor with different gases at $1173 \mathrm{~K}$.

Our observations are also consistent with previous studies in which increased swelling of low porosity samples was attributed to the predominance of iron whisker formation during metallization of wustite under chemically controlled conditions. ${ }^{10)}$ On this basis, high swelling of low porosity samples particularly MTN lumpy sample can be most likely attributed to iron whisker formation. However no additional data was available to further confirm it. The study implies that high porosity iron feed samples would produce less fines due to less swelling.

Figure 18 shows the changes in average compressive strength of iron oxides as the samples are progressively reduced while moving from upper to lower section of the reactor. Compressive strength data is normalized by considering compressive strength of iron oxide before reaction to be $100 \%$. Figure 18 shows that the crushing strength of oxides changes rapidly during initial $10 \%$ reduction and continues to decrease up to $30 \%$ reduction. Subsequently, after $50 \%$ reduction, compressive strength of iron oxide continues increasing progressively. The minimum crushing strength of iron compacts around $30 \%$ reduction could be contributed to lattice distortion due to transformation from hematite to magnetite which is generally known to be responsible for normal swelling and lower strength, while subsequent strength improvements could be related to increasing presence of metallic iron phase. ${ }^{30)}$

The results have serious implications on the efficiency of COREX furnace. If the material has low reduction rate, it would continue to be weaker in the lower part of the reduction shaft where the load by the burden material weight is higher than that in the upper part. This can cause larger amounts of fines generation in the reduction shaft. Therefore, in order to minimize the breakage of the materials in the lower part of the reduction shaft, the reduction rate should be as fast as possible. Therefore, due to adverse effect of slow reaction rates, both reduction rate as well as final extent of reduction should be considered.

Thus, the study clearly demonstrates that the initial porosity of iron oxides would have strong effect on fines generation due to their strong influence on reduction and swelling and hence on weakening in different parts of the reduction shaft. The study shows that generally high porosity iron oxides would promote low swelling, faster reduction and consequently low fines generations. Therefore, the initial porosity of iron oxides could be used to control fines generation during solid state reduction in the reduction shaft and hence to optimize its efficiency.

\section{Conclusions}

Five set of iron compacts including pellets and lumpy ores were reduced under different reacting conditions of gases and temperatures to characterize their reduction and swelling behaviour and their relationship with fines generation. Following conclusions were made.

(1) The study demonstrated that reduction rates of iron oxides was strongly influenced by the initial porosity of the samples such that generally iron ore samples with high porosity indicated fast reduction. However, crack formed in the iron oxides during different stages of reduction could also modify the effect of the initial porosity on the reduction rates due to modification of surface area growth.

(2) In all the tested conditions, low porosity samples indicated high swelling such that all samples displayed normal swelling behaviour i.e. less than $20 \%$ with the exception of one lumpy ore sample with least porosity.

(3) The compressive strength of samples was found to change non-linearly with progressive reduction such that the crushing strength decreased continuously up to $30 \%$ of reduction and increased rapidly after $50 \%$ of the reduction, which can be attributed to increasing sintering of reduced metallic phases.

(4) The laboratory measurements were found to be in agreement with pilot-scale tests such that highest swelling lumpy ore indicated most difficult operating experience. The study implies that high porosity of iron feed samples would facilitate less swelling, faster and high degree of reduction. Consequently, porous iron ore sample would generate less fines during solid state reduction in the reduction shaft. Therefore, as far as the physical properties of iron ore is concerned, the initial porosity can be used to optimize the rate and degree of reduction of iron feed in the reduction shaft and the efficiency of COREX operations.

\section{Acknowledgements}

The authors acknowledge the industrial sponsor, POSCO (Pohang Iron \& Steel Co., Ltd.), Republic of Korea, for supporting this study.

\section{REFERENCES}

1) R. Hauk: Proc. of COREX Symp., SAIMM, South Africa, (1990), 21

2) M. Cho, N. Choi, S.-D. Lee, J. H. Lee, S. Joo and I.-K. Suh: Proc. of ICSTI/Ironmaking Conf., The Iron and Steel Society, Warrendale, PA, (1998), 1211.

3) A. K. Agrawal and T. W. Oshnock: Proc. of Ironmaking Conf., The Iron and Steel Society, Warrendale, PA, (1995), 477.

4) S. C. Panigrahy, M. Jallouli and M. Rigaud: Proc. of Ironmaking 
Conf., Iron and Steel Society, Warrendale, PA, (1984), 233.

5) T. Sharma, R. C. Gupta and B. Prakash: ISIJ Int., 31 (1991), No. 3, 312 .

6) T. Sharma, R. C. Gupta and B. Prakash: ISIJ Int., 32 (1992), No. 7, 812.

7) T. Sharma: ISIJ Int., 34 (1994), No. 12, 960

8) G. Thaning: Iron Steelmaker, 2 (1976), 57.

9) T. Sharma, R. C. Gupta and Prakash: Miner. Eng., 3 (1990), No. 5, 509.

10) R. Nicolle and A. Rist: Metall. Trans. B, 10B (1979), 429.

11) S. Hayashi and Y. Iguchi: ISIJ Int., 43 (2003), No. 9, 1370.

12) J. T. Moon and R. D. Walker: Iron Steelmaker, 1 (1975), 30.

13) J. K. Wright: Trans. Iron Steel Inst. Jpn., 17 (1977), 726.

14) T. Sharma, R. C. Gupta and B. Prakash: ISIJ Int., 32 (1992), No. 12, 1268.

15) A. A. EL-Geassy, M. I. Nasr and M. M. Hessien: ISIJ Int., 36 (1996), No. 6, 640

16) M. I. Nasr, A. A. Omar, M. M. Hessien and A. A. EI-Geassy: ISIJ Int., 36 (1996), No. 2, 164.

17) T. Sharma, R. C. Gupta and B. Prakash: ISIJ Int., 33 (1993), No. 4, 446.

18) S. Taniguchi and M. Ohmi: Mater. Trans. JIM, 19 (1978), 581.
19) S. Taniguchi, M. Ohmi and H. Fukuhara: Trans. Iron Steel Inst. Jpn., 18, (1978), 633.

20) T. Kang, V. Sahajwalla and S. K. Shin: Proc. of 59th Ironmaking Conf., The Iron and Steel Society, Warrendale, PA, USA, (2000), 357.

21) T. Kang, V. Sahajwalla and S. K. Shin: Minprex, Melbourne, Australia, (2000), 11

22) T. Kang: M.Sc. Thesis, The University of New South Wales, Sydney, Australia, (2000).

23) S. M. Nutter and M. N. K. Li: Iron Steelmaker, (1990), April, 65.

24) W. M. McKewan: Trans. AIME, 218 (1960), 2.

$25)$ N. J. Themelis and W. H. Gauvin: Trans. AIME, 227 (1963), 290.

26) E. T. Turkdogan and J. V. Vinters: Metall. Mater. Trans., 2 (1971), 3175 .

27) M. V. C. Sastri, R. P. Viswanath and B. Viswanathan: J. Sci. Ind. Res., 40 (1981), 448.

28) B. B. L. Seth and H. U. Ross: Trans. AIME, 233 (1965), 180.

29) T. L. Joseph: Metals Technology, AIME Technical Publication, 688, (1936), 1.

30) K. Meyer: Pelletizing of Iron Ores, Springer-Verlag, Berlin, (1980), 159. 